\title{
Mechanical Properties of N,N,N-trimethylchitosan Chloride Films
}

\author{
Douglas de Britto, Sérgio P. Campana-Filho \\ Instituto de Química de São Carlos, USP \\ Odilio B.G. de Assis \\ Embrapa Instrumentação Agropecuária, São Carlos, SP
}

\begin{abstract}
Films of chitosan and N,N,N-trimethylchitosan were cast from aqueous solutions. Chitosan was dissolved in 1\% acetic acid while deionized water was the solvent for N,N,N-trimethylchitosan chloride. The resulting films presented different mechanical behaviors as evaluated by DMTA. The film of chitosan exhibited an elastic-type behavior while that of N,N,N-trimethylchitosan was typically viscous. No glass transition temperatures were observed; however, a discrete thermal transition was detected at $25{ }^{\circ} \mathrm{C}$ in the case of the
\end{abstract}

$\mathrm{N}, \mathrm{N}, \mathrm{N}$-trimethylchitosan.

Keywords: Chitosan, N,N,N-trimethylchitosan, mechanical properties.

\section{Introduction}

Chitosan is a biocompatible, biodegradable and non-toxic linear polymer, commercially obtained by deacetylation of chitin, which is an abundant polysaccharide extracted from the shells of shrimps and crabs. Due to its particular properties, chitosan has been currently used in a number of applications such as in the pharmaceutical industry ${ }^{[1]}$, in wastewater separation membranes ${ }^{[2]}$ as well as in edible protective coatings on minimally processed food ${ }^{[3,4]}$. In particular, the use of chitosan films and coatings to extend shelf life and improve the quality of fruits and vegetables has received considerable attention in the last few years ${ }^{[5-7]}$.

Chitosan films can be easily obtained by casting its solution on a desired surface and allowing spontaneous solvent evaporation. It is well known that chitosan exhibits good anti-microbial properties and therefore its films can also provide an effective barrier against microbial and fungi deterioration $^{[8]}$, being a supplementary means to control morphological and physicochemical changes mainly in natural products. To be useful as coating, however, the chitosan film should also exhibit adequate mechanical properties. The tensile strength of the chitosan films lay in the range 50-66 $\mathrm{MPa}$ depending on the degree of deacetylation ${ }^{[9]}$ and on the solvent used in the preparation of the chitosan solution employed in the casting step ${ }^{[10]}$. Hwang et al. ${ }^{[11]}$ have reported that the tensile strength of chitosan films increases with increasing molecular weight while the film elongation seems to be unaffected by the polymer molecular weight. Another study showed that the film strength ranged from 49.6 to $59.4 \mathrm{MPa}$ depending on the drying conditions employed after wet casting ${ }^{[12]}$.
Recently, Miranda et a ${ }^{[13]}$. have showed that the tensile strength, the elongation and the water permeability of chitosan films are strongly affected by the presence of plasticizers, such as poly(ethylene glycol), glycerol and sorbitol, and by their association with methylcelullose to form composite films.

Chitosan is a $\beta(1 \rightarrow 4)$ aminoglucan soluble in aqueous media only if $\mathrm{pH} \leq 5.5$ since the presence of enough acid promotes the protonation of its amino groups and confers a polycationic character to the polymer. Thus, the dilute aqueous solutions of hydrochloric, acetic, lactic, and citric acids are commonly used as chitosan solvents. The use of such solvents, however, represents serious drawbacks to the mechanical properties of the chitosan films and to its biocompatibility, which is reduced at low $\mathrm{pH}$. Also, the acid medium favors the occurrence of degradation reactions, another serious drawback mainly when using chitosan for food protection. To overcome these problems the use of water-soluble chitosan derivatives has been proposed ${ }^{[14]}$. An interesting approach to prepare water soluble chitosan derivatives is the introduction of permanent positive charges in the polymer chains, imparting to them a cationic polyelectrolyte character independently of the $\mathrm{pH}$. It can be accomplished, for instance, by the quaternization of the nitrogen atoms of the amino groups of chitosan to result in $\mathrm{N}, \mathrm{N}, \mathrm{N}$-trialkylchitosan. Thus, N,N,N-trimethylchitosan results from the extensive methylation of chitosan, a reaction which is carried out in suspension of N-methyl-2pyrrolidone with excess of iodomethane and in the presence of sodium iodide and sodium hydroxide ${ }^{[15-17]}$.

The thermal stability of N,N,N-trimethylchitosan films

Autor para correspondência: Sérgio P. Campana-Filho, Instituto de Química de São Carlos, USP, Av. Trabalhador São-carlense 400, CEP: 13560-970, São Carlos, SP. E-mail: scampana@iqsc.usp.br 
has been recently reported ${ }^{[17]}$ and this work describes the mechanical properties evaluation of cast $\mathrm{N}, \mathrm{N}, \mathrm{N}-$ trimethylchitosan chloride films.

\section{Experimental}

Chitosan (Fluka, MW 400,000 g/mol) was dissolved in lactic acid (1M) by stirring overnight, followed by filtration under positive pressure to eliminate insoluble matter. The chitosan was precipitated by adding concentrated $\mathrm{NaOH}$ to the resulting solution and the purified polysaccharide was recovered after extensive washing. The resultant chitosan was dried at room temperature and milled to particles of average size lower than $125 \mu \mathrm{m}$.

The N,N,N-trimethylchitosan was prepared as described elsewhere ${ }^{[16,17]}$ by reacting $3.0 \mathrm{~g}(0.0175 \mathrm{~mol})$ of chitosan suspended in $\mathrm{N}$-methyl-2pyrrolidone $/ \mathrm{NaOH}(14.5 / 1 \mathrm{v} / \mathrm{w})$ with $8.6 \mathrm{~cm}^{3}$ of $\mathrm{CH}_{3} \mathrm{I}$ in the presence of $\mathrm{NaI}(3.62 \mathrm{~g})$.

The ${ }^{1} \mathrm{H}$ NMR spectra of chitosan and N,N,Ntrymethylchitosan were acquired at $353 \mathrm{~K}$ by using a $200 \mathrm{MHz}$ spectrometer (Bruker AC200). For these analysis the samples of chitosan and

$\mathrm{N}, \mathrm{N}, \mathrm{N}$-trimethylchitosan were dissolved in $\mathrm{D}_{2} \mathrm{O} / \mathrm{HCl}(100 /$ $1 \mathrm{v} / \mathrm{v}$ ) and in $\mathrm{D}_{2} \mathrm{O}$, respectively, at a concentration of $10 \mathrm{~g} / \mathrm{L}$. The parameters for the acquisition of the NMR spectra were as follow: a pulse of $90^{\circ}$, corresponding to a pulse width of $8.2 \mu \mathrm{m} ; \mathrm{LB}=0.3 \mathrm{~Hz} ; \mathrm{NS}=16$. The average degrees of acetylation $(\overline{D A})$ and quaternization $(\overline{D Q})$ of chitosan and $\mathrm{N}, \mathrm{N}, \mathrm{N}-$ trimethylchitosan were determined from the ${ }^{1} \mathrm{H}$ NMR spectra as described elsewhere ${ }^{[17]}$.

The films were obtained by casting aliquots of the solutions of chitosan and N,N,N-trimethylchitosan in 1\% acetic acid and water, respectively, on acrylic Preti dishes. The solvent was allowed to evaporate, the resulting films were manually detached from the acrylic surface and then cutted in samples of $55 \times 5 \times 0.03 \mathrm{~mm}$. Dynamic mechanical thermal analysis (DMTA) was carried out using a TA Instrument DMA 2930 in tensile mode, at a heating rate of $5{ }^{\circ} \mathrm{C} \mathrm{min}^{-1}$ and amplitude of cyclic deformation as $20 \mu \mathrm{m}$. In all runs the frequency was $1 \mathrm{~Hz}$.

\section{Results and Discussion}

The ${ }^{1} \mathrm{H}$ NMR spectra of chitosan and its N-methylated derivative are shown in Figure 1. The following signals are observed in the spectrum of chitosan (Fig.1A): $i$ ) signals at $4.5<\delta<5.0$, corresponding to the hydrogen bonded to the anomeric carbon 1 ; ii) signals at $3.4<\delta<4.0$, corresponding to the hydrogens bonded to the carbons atoms $3,4,5$ and 6 of the glucopyranose unit; iii) signal centered at $3.18 \mathrm{ppm}$, which corresponds to the hydrogen bonded to the carbon 2 of the glucopyranose ring and $i v$ ) signal centered at $1.99 \mathrm{ppm}$, which corresponds to the hydrogens of the methyl moieties pertaining to the acetamido groups ${ }^{[16,17]}$. From this spectrum the average degree of acetylation of the purified chitosan was determined as $\overline{D A}=22 \%$. The evidences for the occurrence of N-methylation

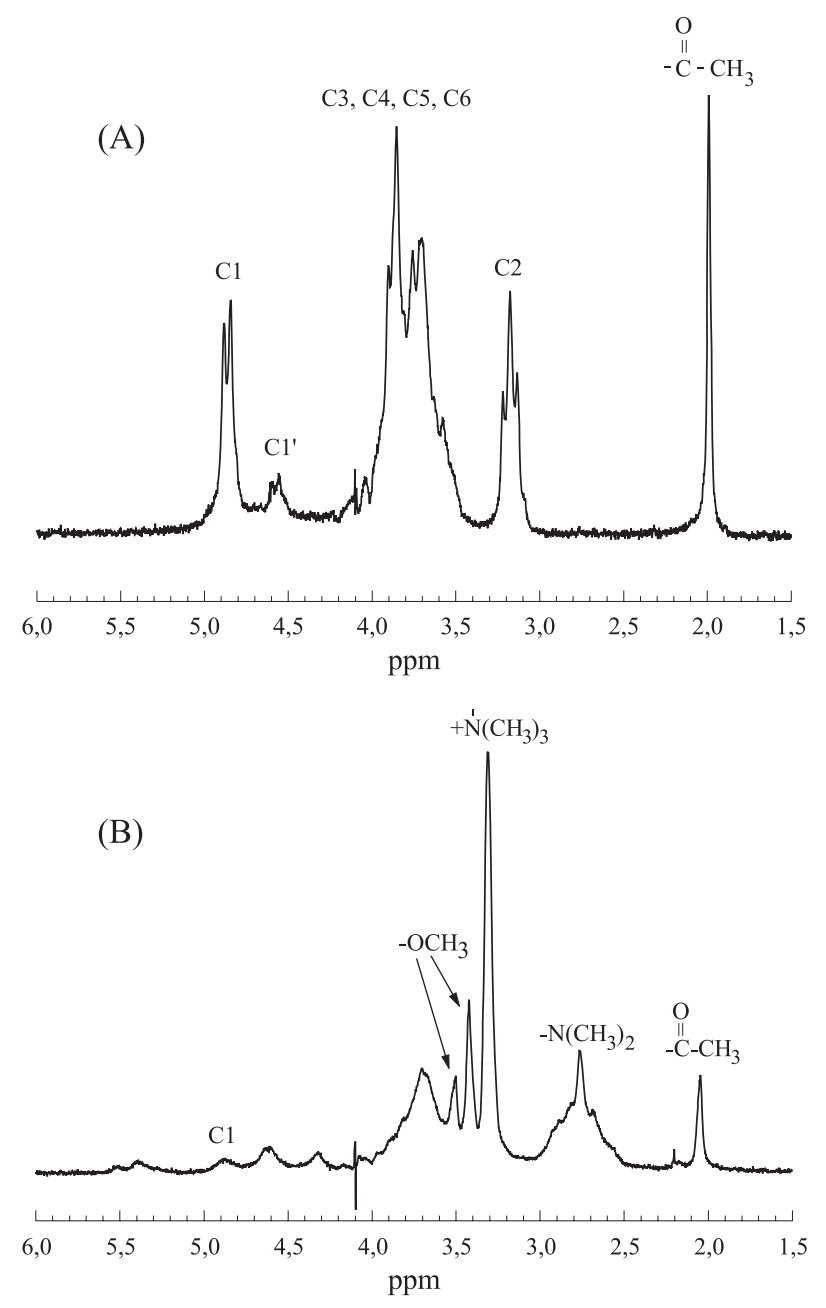

Figure 1. ${ }^{1} \mathrm{H}$ NMR spectra of chitosan $(\mathrm{Cp}=10 \mathrm{~g} / \mathrm{L})$ dissolved in $\mathrm{D}_{2} \mathrm{O} / \mathrm{HC}$ $(100 / 1 \mathrm{v} / \mathrm{v})(\mathrm{A})$, and $\mathrm{N}, \mathrm{N}, \mathrm{N}$-trimethylchitosan $(\mathrm{Cp}=10 \mathrm{~g} / \mathrm{L})$ dissolved in $\mathrm{D}_{2} \mathrm{O}(\mathrm{B})$.

are seen in the region $2.47<\underline{\delta}<3.37$ (Fig.1B) but only the signal centered in $3.31 \mathrm{ppm}$ corresponds to quaternized sites while that signal centered in $2.76 \mathrm{ppm}$ is attributed to N,N-dimethylated sites. This spectrum also shows two signals in the region $3.37<\underline{\delta}<3.56$, both of them corresponding to O-methylated sites. From this spectrum the average degree of quaternization of the $\mathrm{N}, \mathrm{N}, \mathrm{N}$-trimethylchitosan was determined as $\overline{D Q}=37 \%$.

Thermal analysis by DMTA revealed that both polymers, chitosan and N,N,N-trimethylchitosan, present non-linear viscoelastic behaviors but the mode of deformation of chitosan is significantly affected by the quaternization process. Indeed, the stress-strain curves show that the chitosan film exhibits highly elastic behavior with greater elongation while the $\mathrm{N}, \mathrm{N}, \mathrm{N}$-trimethylchitosan film shows a small modulus of elasticity and exhibits a typical viscous behavior (Figure 2). The chitosan film instantaneously respond to the applied stress performing an elastic strain of approximately $2.5 \%$, therefrom the deformation regime changed but no breaking in the film was observed over the stress range tested. The literature reports that shear strain ranging from 50 to $150 \mathrm{MPa}$ correspond to deformation in the order of $8 \%$ to $40 \%{ }^{[19]}$. In this way, several factors affect the interchain interactions and the chitosan 


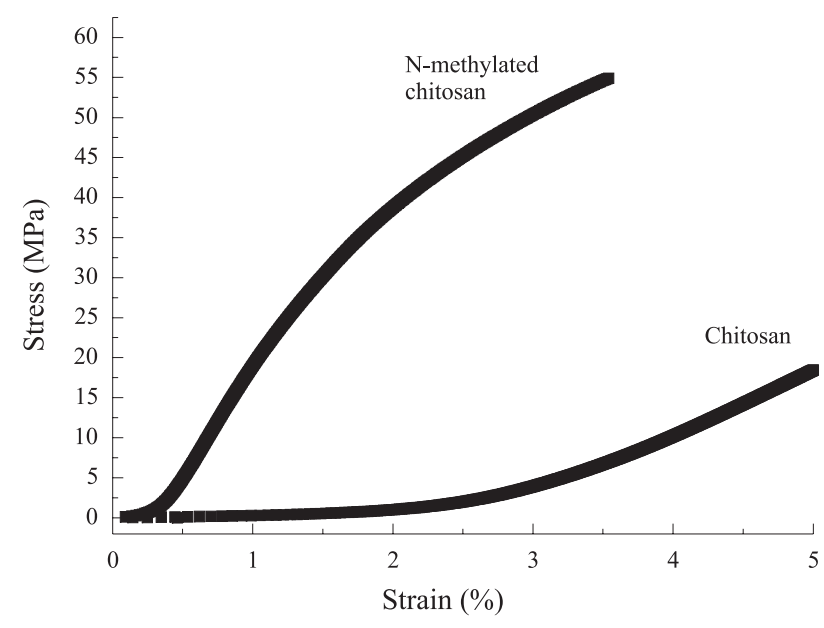

Figure 2. Stress-strain curves for chitosan and N,N,N-trimethylchitosan at room temperature.

dynamic rheology during stretching, such as the molecular weight, degree of deacetylation, water content and crystallinity of the polymer.

The N,N,N-trimethylchitosan film has a small modulus of elasticity under a typical viscous behavior, what means that a greater stress has to be imposed to result in the same elongation as that achieved by chitosan. The fracture of the film takes place when the elongation approaches $3.5 \%$.

The glass transition temperature $\left(\mathrm{T}_{\mathrm{g}}\right)$ is one of the most important criterion to characterize the mechanical properties of a polymer. The $\mathrm{T}_{\mathrm{g}}$ of chitosan and chitin are yet to be precisely characterized but it is known that due to the existence of an extensive number of inter and intrachain hydrogen bonds, the degradation in these polymers occurs before attaining their glass transition temperature. Nevertheless some works ${ }^{[19,20]}$ pointed out estimative values of $205^{\circ} \mathrm{C}$ and $236{ }^{\circ} \mathrm{C}$ for the $\mathrm{T}_{\mathrm{g}}$ of chitosan and chitin, respectively. The reduction in the number of hydrogen bonds, via the introduction of substituent groups in the hydroxyl moieties of these polymers, can decrease their $\mathrm{T}_{\mathrm{g}}$. Thus, the $\mathrm{T}_{\mathrm{g}}$ of acyl-derivatives of chitosan can range from -10 to $-42{ }^{\circ} \mathrm{C}$, depending on the acyl substituent and degree of substitution $^{[21]}$. Owing to the ionic feature of polyelectrolytes, $\mathrm{T}_{\mathrm{g}}$ is expected to shift to higher temperatures when compared to non-charged polymers, due to the strongest electrostatic interchain interactions. The curves relating the storage modulus (E') of chitosan and N,N,N-trimethylchitosan films with the temperature are seen in Figure 3.

A continuous decrease of E' with increasing temperature is observed for chitosan film while the curve corresponding to the N,N,N-trimethylchitosan film shows an important decrease of E' close to $25^{\circ} \mathrm{C}$. That is followed by a recovering of initial values for temperature higher than $100{ }^{\circ} \mathrm{C}$. Whether only the decrease of E' were observed, it could precisely describe the $\mathrm{T}_{\mathrm{g}}$. However, the behavior exhibited by the N,N,N-trimethylchitosan film suggests an important role for the water contained into the polymeric net. Indeed, as a second sweep was performed a small shift to lower temperature values was noticed on E' and, additionally, an important decrease of the area under the peak was clearly observed. Since the N,N,N-

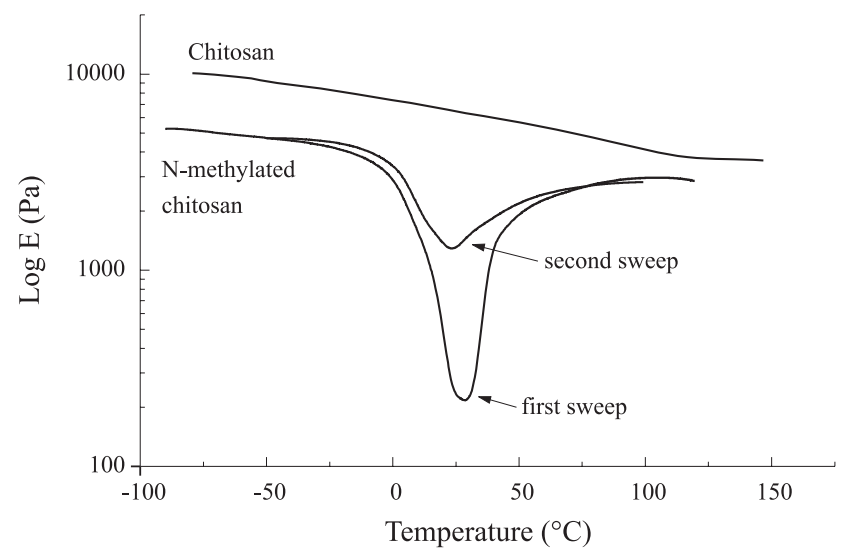

Figure 3. Storage modulus versus temperature for chitosan and N,N,Ntrimethylchitosan.

trimethylchitosan chloride has a strong hydrophilic character, the presence of adsorbed water should be an important factor in the decreasing of the interchain interactions, weakening the mechanical properties by means of a plastifying effect ${ }^{[19]}$. Nevertheless, the actual Tg value remains uncertain.

\section{Conclusions}

The N-methylation of chitosan promotes important changes in its mechanical properties. DMTA analysis showed that films of chitosan and N,N,N-trimethylchitosan presented viscoelastic behaviors, however the former exhibited highly elastic behavior with greater elongation while the latter has a small modulus of elasticity and a typical viscous behavior. The curves of storage modulus versus temperature of the films of chitosan and N,N,N-trimethylchitosan also pointed to different behaviors. The N,N,N-trimethylchitosan films presented a discrete transition at $25^{\circ} \mathrm{C}$ whose intensity seems to depend on the water content.

\section{Acknowledgements}

The authors are grateful to CAPES, CNPq, Embrapa and FAPESP.

\section{References}

1. Ko, J.A.; Park, H.J.; Hwang, S.J.; Park, J.B.; Lee, J.S. - Int. J. Pharm. 249[1-2], p.165-174, (2002).

2. Pietrelli, L.; Xingrong, L. - Annali di Chimica. 94[5-6], p.389-398, (2004).

3. Agulló, E.; Rodríguez, M.S.; Ramos, V.; Albertengo, L. Macromolecular Bioscience, 3[10], p. 521-530, (2003).

4. Assis, O.B.G.; Pessoa, J.D.C. - Braz. J. of Food Sci. Tech., 17[1], p.17-22, (2004).

5. Worrell, D. B.; Carrington, C. M. S.; Huber, D.J. - Post. Biol.Technol., 25, p.33-40, (2002). 
6. Shahidi, F.; Arachchi, J.K.V.\& Jeon, Y-J. - Trends in Food Scien. \& Techn., 10, p.37 (1999).

7. Assis, O.B.G., Leoni, M.A. - Biotec. Ciência e Desenvolvimento. 30, p.33-38, (2003).

8. Möller, H., Grelier, S., Pardon, P., Coma, V. - J. Agric. Food Chem. 52, p. 6585-6591 (2004).

9. García, M.A.; Pinotti, A.; Martino, M.N.; Zaritzky, N.E. Carbohyd. Polym., 56, p.339-345, (2002).

10. Bégin, A.; Calsteren, M.-R.V. - Inter. J. of Biolog. Macromol., 26, p.63-67, (1999).

11. Hwang, K.T.; Kim, J.T.; Jung, S.T.; Cho, G.S.; Park, H.J. - J. Appl. Polym. Sci., 89, p.3476-3484 (2003).

12. Srinivasa, P.C.; Ramesh, M.N.; Kumar, K.R.; Tharanathan, R.N. - J. Food Engineering, 63, p.79-85, (2004).

13. Miranda, S.P.; Garnica, O.; Lara-Sagahon, V.; Cárdenas, G. - J. Chil. Chem. Soc., 49, p.173-178, (2004).
14. Lazaridou, A.; Biliaderis, C.G. - Carbohyd. Polym., 48, p.179-190, (2002).

15. Britto,D. PhD Thesis, IQSC-USP, São Carlos, SP., 2003, 146p.

16. Curti,E.; Britto,D.; Campana Filho, S.P. - Macromol. Bioscience, 3, p.571-576 (2003).

17. Britto,D., Campana Filho,S.P. - Polym. Degrad. and Stability, 84, p.353-361, (2004).

18. Rathke, T.D.; Hudson, S.M. - J.M.S - Review Macromol. Chem. and Phys., v.C34, p.375-437, (1994).

19. Sakurai, K; Maegawa,T.; Takahashi, T. Polymer, 41, p.7051-7056, (2000).

20. Kim, S.S.; Kim, S.H.; Lee, Y.M.; - J. Polym. Sci.: Part B, Polym. Phys., 34, p.2367-2374, (1996).

21. Zong, Z.; Kimura, Y.; Takahashi, M.; Yamani, H. - Polymer, 41, p.899-906, (2000).] 